\title{
1997 October Event(s) in the Crab Pulsar
}

\author{
D. C. Backer
}

Astronomy Department, University of California, Berkeley, CA, USA

\begin{abstract}
In October 1997 daily monitoring observations of the Crab pulsar at $327 \mathrm{MHz}$ and $610 \mathrm{MHz}$ with an $85 \mathrm{ft}$ telescope in Green Bank, WV showed a jump in the dispersion by $0.12 \mathrm{~cm}^{-3} \mathrm{pc}$. Pulses were seen simultaneously at both old and new dispersions for a period of days. In the months before this event faint ghost emission, a replica of the pulse, was detected with a nearly frequency independent delay that quadratically diminished to zero. There was also a curious shift in the phase, a slowdown, at all frequencies at the time of the dispersion jump. I attribute most of these phenomena to the perturbing optics of a plasma prism that is located in the filamentary interface between the synchrotron nebula and the supernova ejecta and which crosses the line of sight over a period of months. The required density, scale length and velocity are reasonable given detailed HST and previous observations of these filaments.
\end{abstract}

\section{Introduction}

In 1975 the dispersion measure, rotation measure and scattering of the Crab pulsar displayed an extreme level of activity (Lyne \& Thorne 1975; Isaacman \& Rankin 1977). These disturbances in the propagation of radiation from the pulsar were ascribed to thermal plasma associated with the Crab nebula. In general the variations of the Crab pulsar's dispersion measure are larger than that expected for the interstellar medium, and are thus likely the result of nebular material (Backer et al. 1993). In recent years the dispersion measure and scattering have been undergoing a new series of large variations (Backer \& Wong 1996). Column density variations of $0.1 \mathrm{pc} \mathrm{cm}^{-3}$ over time scales of months are seen. If one places these variations in the filamentary web surrounding the optical synchrotron nebula, then the likely transverse velocity of the pulsar-Earth line of sight relative to the plasma $\sim 200 \mathrm{~km} \mathrm{~s}^{-1}$. In this case one can estimate that the characteristic density of the perturbations is $\sim 2500 \mathrm{e} \mathrm{cm}^{-3}$ and the typical transverse length is $\sim 10^{14} \mathrm{~cm}$. This density is reasonably consistent with density estimates of the filaments from optical line measurements (Hester et al. 1996, Sankrit et al. 1998) that have a linear resolution which is several orders of magnitude larger, $\sim 10^{16} \mathrm{~cm}$. There has also been a unique cluster of glitches in the rotation of the star during the past few years (Wong, Backer \& Lyne 1999).

In October 1997 amidst this era of large variations of dispersion measure and other plasma propagation parameters as well as internal "seismic" events, a dispersion measure "glitch" (sudden change in less than one week) of 0.12 
$\mathrm{cm}^{-3} \mathrm{pc}$ was noticed in measurements both at the NRAO Green Bank site and at the University of Manchester Jodrell Bank site (see Smith \& Lyne in this volume). These observatories have small dedicated telescopes for pulsar monitoring. In fact, during the glitch event pulses were simultaneously detected at both dispersions. Subsequently Smith \& Lyne found that for about two months prior to the dispersion event a faint replica ("ghost") of the pulsed emission following the main pulse and interpulse components was detected in the Jodrell Bank $610-\mathrm{MHz}$ data. Receding ghost components are observed a few months after the dispersion glitch. The Green Bank observations confirmed the $610-\mathrm{MHz}$ ghost components and further provided detections at $327 \mathrm{MHz}$. The phase of the ghost components slowly converged to that of the regular emission a week before the dispersion glitch.

While many of the phenomena can be explained by an imperfect plasma lens passing through the line of sight at the distance of the Crab nebula filaments, the occurence of an unusual spindown of the neutron star phase during the dispersion glitch, and a small but more conventional spinup glitch of the star two months after the dispersion glitch provide arguments for consideration of plasma propagation in the vicinity of the star. The ghost of the main pulse and that of the interpulse are not always identical which adds further confusion to interpretation. In this brief report, the $327-\mathrm{MHz}$ sequence of events is described. This is followed by a discussion of the optics that might give rise to these events. A full report is being submitted for journal publication.

\section{2. $\quad 327-\mathrm{MHz}$ event}

I will refer to the pulse profiles in Figure 1 before and after the dispersion glitch as "old" and "new" respectively. The old pulse peak amplitude is reasonably steady at the start, then has two peaks centered on 1997 day 265 and day 275 , and then fades away by day 300 . The new pulse is shifted in phase by 0.15 $(5 \mathrm{~ms})$ and has amplitudes which rise steadily from day 290 to day 365 . The new pulses appear faint owing to a large pulse broadening that I will attribute to severe multipath propagation in the extra dispersion medium. The ghost component of the main pulse appears at phase 0.4 around day 250 and moves along a quadratic path toward the main pulse at phase 0.28 around day 275 . The ghost of the interpulse follows a similar path. The peak intensities are typically $1-2 \%$ times that of the corresponding pulse components. Note that during days 285 to 299 there is emission present simultaneously from old and new pulses. I interpret this in terms of two propagation paths from the pulsar in the next section.

Extrapolation of the old pulse phase to day 320 leads to an offset between new and old pulses of $5.1 \mathrm{~ms}$ at $327 \mathrm{MHz}$. The multipath propagation delay from both new and old pulses have been removed in this estimate. The corresponding number at $610 \mathrm{MHz}$ is $2.1 \mathrm{~ms}$. These two delays are not consistent with the cold plasma, quadratic dispersion law. There appears to be a 0.9 -ms achromatic delay. The source of this delay is actually just detectable in the fading old pulse emission: in Figure 1 the peak of the main and interpulse is rapidly drifting to the right during days 290 to 299. Most of this drift is achromatic; i.e., it is seen at both $327 \mathrm{MHz}$ and $610 \mathrm{MHz}$. Achromatic variations of pulse arrival time 


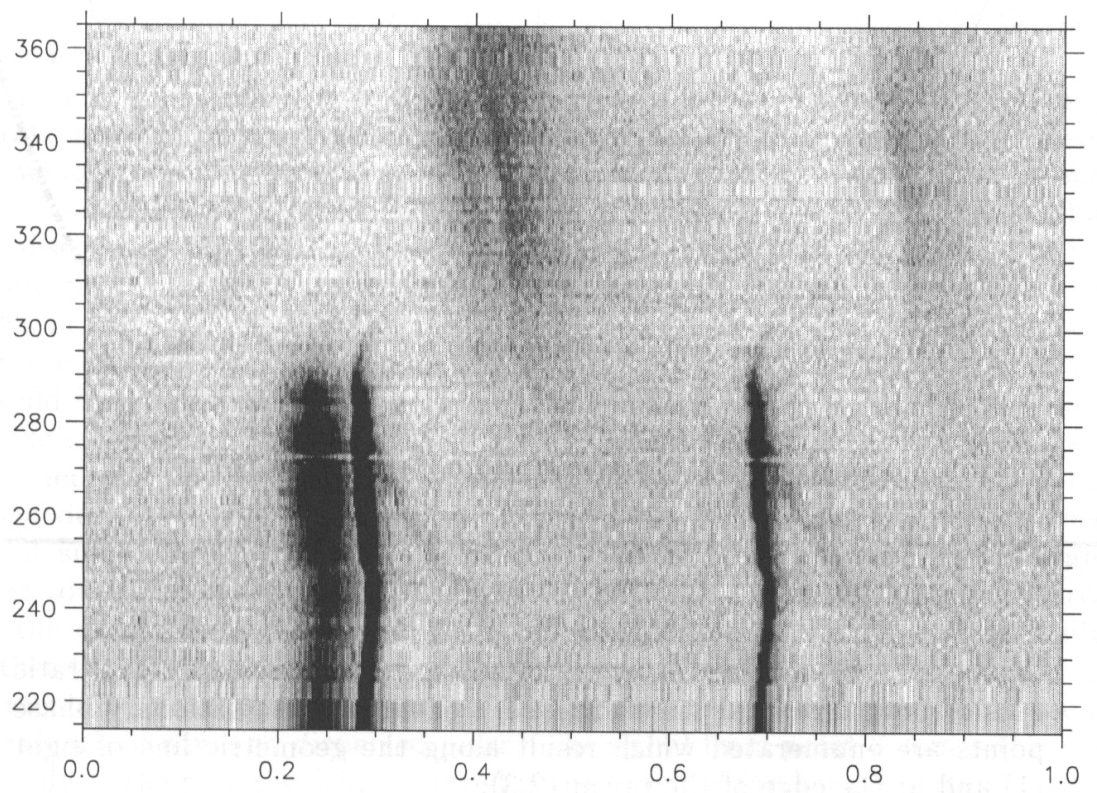

Figure 1. Record of the pulse shapes at $327 \mathrm{MHz}$ during the interval of 1997 day 225 to 1997 day 365 . Pulse fraction is on the abscissa and day number on the ordinate. The pulse intensity, highly saturated to show weak emission, is plotted on grey scale. The pulse consists of precursor, main pulse and interpulse components (from left to right). 


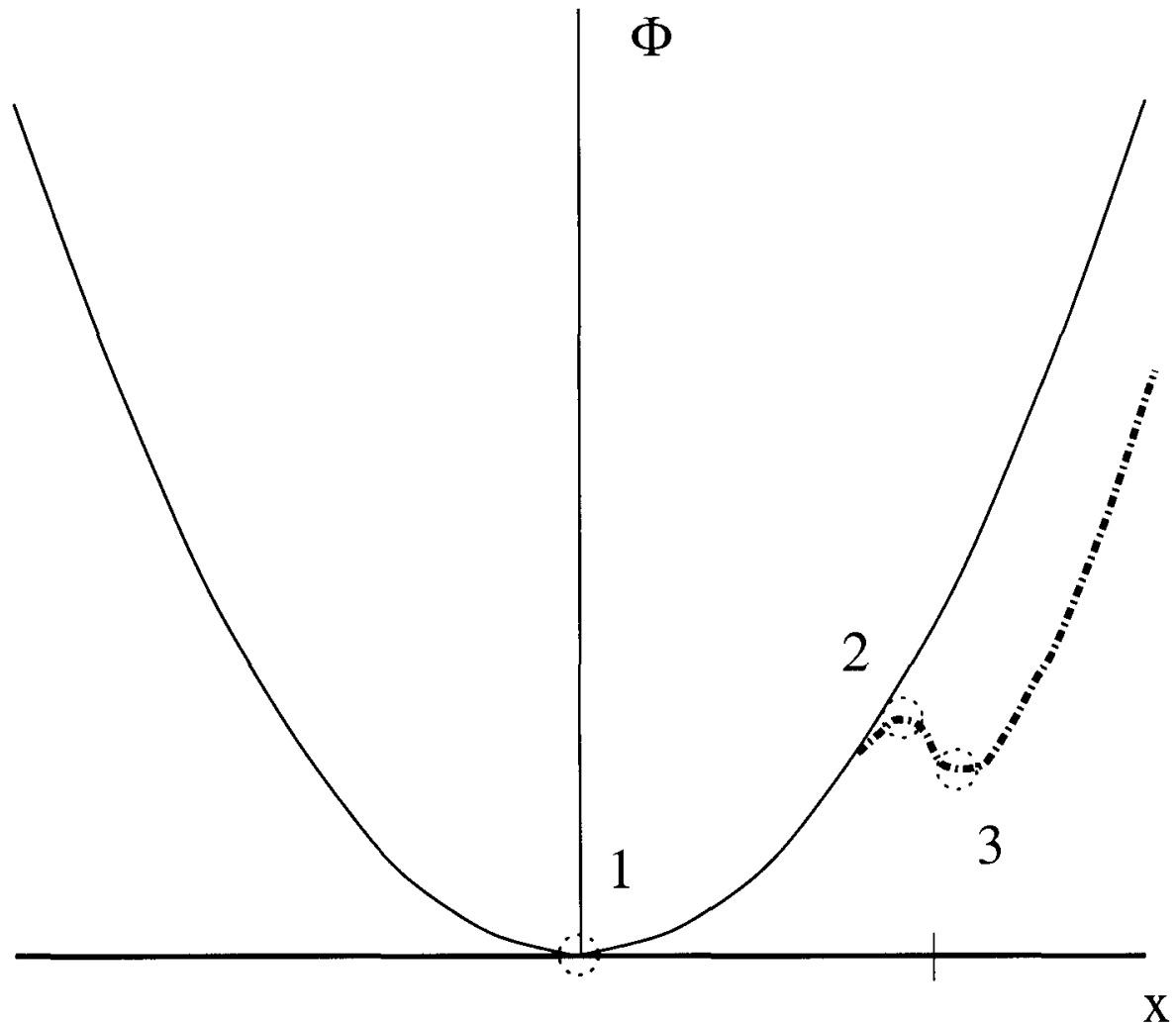

Figure 2. Wave phase through the plasma prism including quadratic Fresnel bowl term and the leading edge of the prism. Stationary phase points are enumerated which result along the geometric line of sight (1) and at the edge of the prism $(2,3)$.

are most naturally interpreted as seismic events internal to the neutron star. Thus just at the time of this dispersion glitch with emission seen along two lines of sight simultaneously, there appears to be a spindown of the pulsar of rather large amplitude - large with respect to the timing noise in this pulsar which is characterized by a random walk in frequency. I will proceed with discussing the ghost component and dispersion glitch in terms of propagation through a plasma prism in the filamentary interface of the Crab nebula, and leave the "coincidence" of an internal effect aside. A final remark here, in admittedly weak support of ignoring the nominally internal event, is that the pulsar has been extremely active with many spin glitches over the 1996-1998 interval.

\section{Plasma Prism Model}

The dispersion glitch and the simultaneous appearance of radiation along nominal dispersion (old) and extra dispersion (new) paths can readily be explained 
by a wedge of plasma moving into the line of sight. The extra dispersion largely disappears after 250 days. The start of the steady decline can be seen in the drifting location of the new pulse in Figure 1 between days 300 and 365 . Thus the dispersion changes can be explained as a plasma prism passing through the line of sight and oriented so that one observes a fairly abrupt change of dispersion. The refractive property of a simple uniform density plasma prism, with its cold plasma index of refraction less than unity, is such as to bend the new pulse into the line of sight and allow simultaneous observation of the old and new pulse. From the observed gradient in dispersion I estimate a refraction angle of $1 \mu \mathrm{rad}$ at $327 \mathrm{MHz}$. Refraction of this magnitude at a point $2 \mathrm{pc}$ along the line of sight from the pulsar to Earth would lead to a small time delay of $0.2 \mathrm{~ms}$ at $327 \mathrm{MHz}$. Of course this geometrically delayed path would also have the new dispersion delay.

Figure 2 presents the optics of this plasma prism model more explicitly by taking a cut of the total wave phase as the wave exits the plasma prism. The wave phase consists of a geometric part, a cut through a quadratic 'Fresnel bowl', and the plasma prism part shown to the right of the line of sight. What one observes at any instant is signal from the stationary phase points. With a plasma prism, as with a gravitational lens, one sees an odd number of signal paths, in this case 3 . Path 1 is the unperturbed geometric line of sight. Path 2 is on the leading edge of the prism and path 3 from within the prism. Thus path 2 would have approximately the same dispersion as path 1 while 3 would have the excess dispersion of the prism. The flux one observes from each stationary point depends on the size of the stationary phase patch, or more explicitly the second derivative of the phase (e.g. Clegg et al. 1998). For the unperturbed line of sight, the size is that of the first Fresnel zone. As the line of sight moves toward the prism's edge, the location of 2 and 3 changes roughly linearly, and the excess delay along this path will change approximately quadratically. Further this geometric path delay effect is approximately achromatic. Thus we can identify point 2 with the ghost component and point 3 with the simultaneous appearance of new and dispersed pulse. The signal from point 3 comes through the body of the prism which, as stated above, has the further property of excess multipath propagation. This effect can lead to the difficulty of seeing the third pulse during days when the ghost pulse is seen (245-275).

\section{Conclusion}

I have presented the $327-\mathrm{MHz}$ Crab pulsar data from late 1997 . These show a number of events - ghost components and dispersion glitch and spindown. The principal features can be explained by propagation through a plasma prism that takes several months to drift through the line of sight. The physical parameters of the prism - size of $3 \times 10^{14} \mathrm{~cm}$ and density of $1200 \mathrm{e} \mathrm{cm}^{-3}$ - are reasonably consistent with those determined by forbidden line observations of the filamentary gas surrounding the optical synchrotron nebula. The density is possibly high given the small length scale in relation to the filamentary structure discussed by Hester et al. 1996 and Sankrit et al. 1998. The density can be reduced by choosing a sheet or pencil geometry that extends into the plane 
of the sky by larger scale than what is detected in the transverse direction (see similar argument for interstellar tiny HI structures in Heiles 1997).

Acknowledgments. I thank the NRAO staff at the Green Bank site who have helped to maintain the pulsar monitoring telescope program there, Graham Smith and Andrew Lyne for many discussions on these still puzzling events, and Berkeley students Tony Wong and Jay Valanju who assisted in data analysis.

\section{References}

Backer, D., et al. 1993, ApJ, 404, 636

Backer, D., \& Wong, T. 1996, ASP CS, 105, 87

Clegg, A., Fey, A., \& Lazio, J. 1998, ApJ, 496, 253

Heiles, C. 1997, ApJ, 491, 193

Hester, J., et al. 1996, ApJ, 456, 225

Isaacman, R., \& Rankin, J. 1977, ApJ, 214, 214

Lyne, A., \& Thorne, D. 1975, MNRAS, 172, 97

Sankrit, R., \& Hester, J. 491, ApJ, 1997, 796

Wong, T., Backer, D., \& Lyne, A. 1999, in preparation 